\title{
Characteristics of Non-Small Cell Lung Cancer Located in the Right Middle Lobe According to a Retrospective Study of Recurrence and Prognosis ${ }^{*}$
}

\author{
Katsuhiko Shimizu\#, Yuji Hirami, Riki Okita, Shinsuke Saisho, Takuro Yukawa, Ai Maeda, \\ Koichiro Yasuda, Masao Nakata \\ Department of General Thoracic Surgery, Kawasaki Medical School, Kurashiki, Japan. \\ Email: "kshimizu@med.kawasaki-m.ac.jp
}

Received May $11^{\text {th }}, 2012$; revised June $11^{\text {th }}, 2012$; accepted June $20^{\text {th }}, 2012$

\begin{abstract}
Background: Some studies have suggested that among all cases of lung cancer, the outcome of lung cancer located in the right middle lobe (RML) is the worst. However, with the advances in the diagnosis and treatment methods of lung cancer over the last couple of decades, we investigated whether the prognosis of primary lung cancer located in the RML still remains inferior to that of lung cancer arising from other lobes. Methods: Between July 2003 and December 2011, 505 consecutive patients with non-small cell lung cancer (NSCLC) underwent surgical resection at our institution. Of these, 32 patients $(6.3 \%)$ had tumors arising from the RML. Results: The rate of incomplete resection was higher for cancer located in the RML than that for cancer arising from other lobes. Significant associations were noted between cancer located in the RML and the rate of lymph node metastasis and initial locoregional recurrence. Multivariate analysis identified lymph node metastasis and location in the RML as independent risk factors influencing the recurrence-free survival $(p=0.006)$, although location in the RML was not extracted as an independent risk factor influenceing the overall survival $(p=0.060)$. Conclusion: Despite the recent advances in the treatment of lung cancer, evaluation of complete resection revealed that the outcome of cancer located in the RML is still the worst among cancer of all the lobes. Further early diagnosis and adjuvant therapy are needed for improving the prognosis of cancer located in the RML.
\end{abstract}

Keywords: Right Middle Lobe; Non-Small Cell Lung Cancer; Prognosis

\section{Introduction}

The right middle lobe (RML) is the smallest lobe of the lungs. Primary lung cancer originating from the RML is much less common than that arising from the other lobes, and is estimated to account for $3.8 \%-6.7 \%$ of all lung cancers [1-3]. Some studies have reported that among all lung cancers, cancers arising in the RML have the worst outcomes [1-3]. On the other hand, a recent report demonstrated that the outcome of cancer located in the RML was no worse than that of cancers arising from other lobes of the lung [4]. However, all of the earlier reports were published between 15 and 35 years ago. Over the decades since, marked advances have been made in the diagnosis and treatment of lung cancer, and we investigated whether primary lung cancer located in the RML may still be associated with a poor prognosis.

\footnotetext{
"Disclosures: The authors have no conflicts of interests to declare.

"Corresponding author.
}

\section{Patients and Methods}

\subsection{Study Population}

We conducted this retrospective study in a total of 505 patients with non-small cell lung cancer (NSCLC) who underwent resection at the Kawasaki Medical School Hospital between July 2003 and December 2011. Of these, 32 patients $(6.3 \%)$ had tumors arising from the RML. Among the 505 patients, 31 cases $(6.1 \%)$ were incomplete resection including exploratory thoracotomy. We excluded these 31 patients, and enrolled the remaining 474 patients in whom complete resection could be accomplished in this study. The histological diagnosis of the tumors was based on the criteria of the World Health Organization, and the TNM stage was determined according to the criteria updated in 2009. This study was conducted with the approval of the institutional Ethics Committee of Kawasaki Medical School. 


\subsection{Statistical Analysis}

Statistical analysis was performed for examining the significances of differences among the groups and the possible correlations between cancer of the RML and other lobes and the clinicopathological features using the Fisher exact test or the $\chi^{2}$ test, as appropriate. An unpaired t-test was used for comparison of the continuous data. Multivariate analyses were performed using a logistic regression analysis. To explore the association between recurrence-free survival (RFS)/overall survival (OS) and cancer of the RML or other lobes, a Kaplan-Meier survival analysis was performed by stratifying significant predictor variables identified using the Cox proportional hazards model. All the statistical analyses were conducted using the SPSS software (Version 17.0; SPSS Incorporation, Chicago, IL). All statistical tests were two-sided, and probability values of $<0.05$ were regarded as denoting statistical significance.

\section{Results}

\subsection{Clinical Characteristics}

The median follow-up period was 32.6 months (range 1 99 months). The rate of incomplete resection was higher for cancer located in the RML than for that arising from the other lobes (Table 1). The characteristics of the 474 patients enrolled in this study are summarized in Table 2. The patients ranged in age from 34 to 90 years (mean, 69.5), and there were 297 men and 177 women.

\subsection{Relation between Location of Cancer and the Clinicopathological Characteristics}

There were 28 (5.9\%) patients with NSCLC located in the RML, consisting of 18 men and 10 women, with a mean age of 68.7 years. The tumors included 20 adenocarcinomas $(71.4 \%)$, seven squamous cell carcinomas (25.0\%), and one large cell carcinoma. Ten patients (35.7\%) had lymph node metastasis. Significant associations of the cancer located in the RML were observed

Table 1. The rate of incomplete resection cases $(n=505)$.

\begin{tabular}{cccc}
\hline \multicolumn{4}{c}{ Resection } \\
\hline Location & Incomplete (A) & Complete (B) & $\mathrm{A} / \mathrm{A}+\mathrm{B}(\%)$ \\
RML & 4 & 28 & 12.50 \\
RUL & 8 & 162 & 4.71 \\
RLL & 6 & 100 & 5.66 \\
LUL & 9 & 110 & 7.56 \\
LLL & 4 & 74 & 5.13 \\
\hline
\end{tabular}

RML vs. other lobes $p=0.124$ ( $p$-value was calculated by Fisher exact test). RML: right middle lobe; RUL: right upper lobe; RLL: right lower lobe; LUL: left upper lobe; LLL: left lower lobe.
Table 2. Patient characteristics enrolled in this study $(n=$ 474).

\begin{tabular}{ccc}
\hline & Number & $\%$ \\
\hline Sex & & \\
Male & 297 & 62.7 \\
Female & 177 & 37.3 \\
Age, mean \pm SD & $69.5 \pm 9.9$ & \\
\hline Histology & & \\
Adenocarcinoma & 326 & 68.7 \\
Squamous cell & 107 & 22.6 \\
Large cell & 24 & 5.1 \\
Adenosquamous & 9 & 1.9 \\
Pleomorphic & 8 & 1.7 \\
\hline Pathological stage & & \\
IA & 216 & 12.0 \\
IB & 125 & 0.6 \\
II (A + B) & 73 & 26.4 \\
III (A + B) & 57 & 15.4 \\
IV & 3 &
\end{tabular}

with the rate of male $(64.3 \%$ vs. $62.6 \%, \mathrm{p}=0.034)$ and the rate of lymph node metastasis $(36.0 \%$ vs. $17.7 \%, \mathrm{p}=$ $0.018)$, but not with the mean of age $(\mathrm{p}=0.703)$, histological type $(\mathrm{p}=0.751)$, or the rate of pleural invasion $(21.4 \%$ vs. $11.9 \%, \mathrm{p}=0.138)$ (Table 3$)$.

\subsection{Relation between the Location of Cancer and the Risk of Recurrence}

One hundred twenty-one (25.5\%) patients developed cancer recurrence after resection. Significant association of cancer located in the RML was observed with the rate of recurrence $(46.4 \%$ vs. $24.2 \%, p=0.009)$. The initial recurrence sites are presented in Table 4 . The initial recurrence site was analyzed: of 474 patients, 53 patients (43.8\%) had locoregional relapse, 76 patients $(62.8 \%)$ had distant relapse, and $8(6.6 \%)$ had both. Significant association of cancer located in the RML was observed with the rate of locoregional recurrence $(25.0 \%$ vs. $10.3 \%$, $\mathrm{p}=0.012$ ), but not with that of distant-site recurrence $(25.0 \%$ vs. $15.5 \%, \mathrm{p}=0.183)$.

\subsection{Prognostic Analysis}

The RFS of patients with cancer located in the RML was significantly worse than that of the patients with cancer arising from the other lobes $(\mathrm{p}=0.018$, according to the 
Table 3. Clinicopathological feature according to the lobe $(n=474)$.

\begin{tabular}{|c|c|c|c|c|c|c|}
\hline Location & RML & RUL & RLL & LUL & LLL & p-value \\
\hline & & & & & & (RML vs. Others) \\
\hline Number & 28 & 162 & 100 & 110 & 74 & \\
\hline \multicolumn{7}{|l|}{$\operatorname{Sex}(\%)$} \\
\hline Male & $18(64)$ & $110(68)$ & $55(55)$ & $68(62)$ & $46(62)$ & 0.034 \\
\hline Female & $10(36)$ & $52(32)$ & $45(45)$ & $42(38)$ & $28(38)$ & \\
\hline Age, mean & 68.7 & 69.8 & 68.4 & 69.9 & 69.8 & 0.703 \\
\hline Histology (\%) & & & & & & 0.751 \\
\hline Squamous cell & $7(25)$ & $31(19)$ & $24(24)$ & $26(24)$ & $19(26)$ & \\
\hline Non-Squamous cell & $21(75)$ & $131(81)$ & $76(76)$ & $84(76)$ & $55(76)$ & \\
\hline Adenocarcinoma & 20 & 117 & 70 & 74 & 45 & \\
\hline Large cell & 1 & 11 & 3 & 4 & 5 & \\
\hline Adenosquamous & 0 & 1 & 2 & 4 & 2 & \\
\hline Pleomorphic & 0 & 2 & 1 & 2 & 3 & \\
\hline \multicolumn{3}{|c|}{ Pathological lymph node status (\%) } & & & & 0.018 \\
\hline $\mathrm{pN} 0$ & $18(64)$ & $141(87)$ & $79(79)$ & $92(84)$ & $55(74)$ & \\
\hline pN1 - 2 & $10(36)$ & $21(13)$ & $21(21)$ & $18(16)$ & $19(26)$ & \\
\hline $\mathrm{pN} 1$ & 3 & 8 & 77 & 10 & 11 & \\
\hline $\mathrm{pN} 2$ & 7 & 13 & 14 & 8 & 8 & \\
\hline \multicolumn{3}{|c|}{ Pathological pleural invasion status (\%) } & & & & 0.138 \\
\hline pl $0-1$ & $22(79)$ & $138(85)$ & $96(96)$ & $94(85)$ & $65(88)$ & \\
\hline pl $2-3$ & $6(21)$ & $24(15)$ & $4(4)$ & $16(15)$ & $9(12)$ & \\
\hline
\end{tabular}

p-value was calculated by $\chi^{2}$ test. RML: right middle lobe; RUL: right upper lobe; RLL: right lower lobe; LUL: left upper lobe; LLL: left lower lobe.

log-rank test; Figure 1(a)). The OS of the patients with cancer located in the RML tended to be worse than that of the patients with cancer arising from the other lobes, although this association was not statistically significant $(\mathrm{p}=0.109$ according to the log-rank test; Figure 1(b)). Multivariate analysis identified lymph node metastasis and location in the RML as independent risk factors influencing the RFS ( $p=0.006$, Table 5(a)). However, multivariate analysis identified only lymph node metastasis as an independent risk factor influencing the OS; location in the RML tended to be independent risk factor, however, the association was not statistically significant $(\mathrm{p}=0.060$, Table 5(b)).

\section{Comment}

There have been several reports focusing on the cancer located in the RML, especially its prognosis. However, all of the earlier reports were published between 15 and 35 years ago. This is the first report focusing on the prognosis of the cancer located in the RML since 2000. In the last couple of decades, much progress has been made in the diagnosis and treatment of lung cancer, such as early diagnosis, less invasive surgery, adjuvant chemotherapy, and molecular-targeted therapy. Therefore, we investigated once again whether primary lung cancer located in the RML may still be associated with a poor prognosis.

In general, the characteristics of lung cancer arising from the middle lobe have been described as follows: 1) high frequency of extralobar invasion or pleural dissemination, and 2) numerous lymphatic drainage sites extending to both the superior and inferior mediastinal zones [4-6]. First, we demonstrated that the rate of incomplete resection was higher for cancers located in the RML than for those located in the other lobes. In our stu$\mathrm{dy}$, incomplete resection was performed in two patients with pleural dissemination, one with mediastinal lymph node involvement, and one residual disease in bronchial 
Table 4. Initial recurrence sites according to the lobe $(n=474)$.

\begin{tabular}{|c|c|c|c|c|c|c|}
\hline Location & RML & RUL & RLL & LUL & LLL & p-value \\
\hline & & & & & & (RML vs. Others) \\
\hline Number & 28 & 162 & 100 & 110 & 74 & \\
\hline \multirow[t]{2}{*}{ Recurrence cases (\%) } & 13 & 35 & 27 & 29 & 17 & 0.009 \\
\hline & $(46.4)$ & $(21.6)$ & $(27.0)$ & (26.4) & $(23.0)$ & \\
\hline \multicolumn{7}{|l|}{ Initial recurrence sites } \\
\hline Locoregional (\%) & $7(25.0)$ & $12(7.4)$ & $13(13.0)$ & $12(10.9)$ & $9(12.2)$ & 0.012 \\
\hline Local & 0 & 4 & 2 & 1 & 3 & \\
\hline Lymph node & $5^{*}$ & $6^{*}$ & $6^{*}$ & $7^{*}$ & $3^{*}$ & \\
\hline Plural dissemination & 2 & 2 & 5 & 4 & 3 & \\
\hline Distant (\%) & $7(25.0)$ & $26(16.0)$ & $16(16.0)$ & $18(16.4)$ & $9(12.2)$ & 0.183 \\
\hline Lung & 3 & 9 & $6^{*}$ & 5 & 4 & \\
\hline Brain & 2 & 6 & 5 & 3 & 1 & \\
\hline Bone & $1^{*}$ & $5^{*}$ & $2^{*}$ & 5 & 0 & \\
\hline Liver & 0 & 5 & 3 & $4^{*}$ & $4^{*}$ & \\
\hline Adrenal & 0 & 1 & 0 & 1 & 0 & \\
\hline Others $^{\#}$ & 1 & 0 & 0 & 0 & 0 & \\
\hline Simultaneous ${ }^{*}$ & $1^{*}$ & $3^{*}$ & $2^{*}$ & $1^{*}$ & $1^{*}$ & \\
\hline
\end{tabular}

p-value was calculated by $\chi^{2}$ test. " gastrointestinal metastasis; RML: right middle lobe; RUL: right upper lobe; RLL: right lower lobe; LUL: left upper lobe; LLL: left lower lobe; " gastrointestinal metastasis.

Table 5. Prognostic analysis.

(a) Multivariate analysis of factors predicting recurrence-free survival $(\mathrm{n}=474)$.

\begin{tabular}{|c|c|c|c|c|}
\hline \multicolumn{2}{|c|}{ Variable } & HR & $95 \% \mathrm{CI}$ & $\mathrm{p}$-value \\
\hline \multirow[t]{2}{*}{ LN metastasis } & negative & 1.00 & & \\
\hline & positive & 5.15 & $3.54-7.49$ & $<0.001$ \\
\hline \multirow[t]{5}{*}{ Location } & RUL & 1.00 & & \\
\hline & RML & 2.53 & $1.30-4.93$ & 0.006 \\
\hline & RLL & 1.13 & $0.67-1.91$ & 0.639 \\
\hline & LUL & 1.22 & $0.74-1.99$ & 0.439 \\
\hline & LLL & 0.83 & $0.46-1.51$ & 0.539 \\
\hline
\end{tabular}

(b) Multivariate analysis of factors predicting overall survival $(n=474)$.

\begin{tabular}{|c|c|c|c|c|}
\hline \multicolumn{2}{|c|}{ Variable } & HR & $95 \% \mathrm{CI}$ & p-value \\
\hline \multirow[t]{2}{*}{ LN metastasis } & negative & 1.00 & & \\
\hline & positive & 2.98 & $2.01-4.42$ & $<0.001$ \\
\hline \multirow[t]{5}{*}{ Location } & RUL & 1.00 & & \\
\hline & RML & 1.94 & $0.97-3.87$ & 0.060 \\
\hline & RLL & 1.19 & $0.69-2.03$ & 0.536 \\
\hline & LUL & 0.99 & $0.59-1.68$ & 0.979 \\
\hline & LLL & 1.18 & $0.67-2.08$ & 0.561 \\
\hline
\end{tabular}

HR: hazard ratio; 95\% CI: 95\% confidence interval; p-value was calculated by log-rank test; LN: lymph node; RML: right middle lobe; RUL: right upper lobe; RLL: right lower lobe; LUL: left upper lobe; LLL: left lower lobe. 


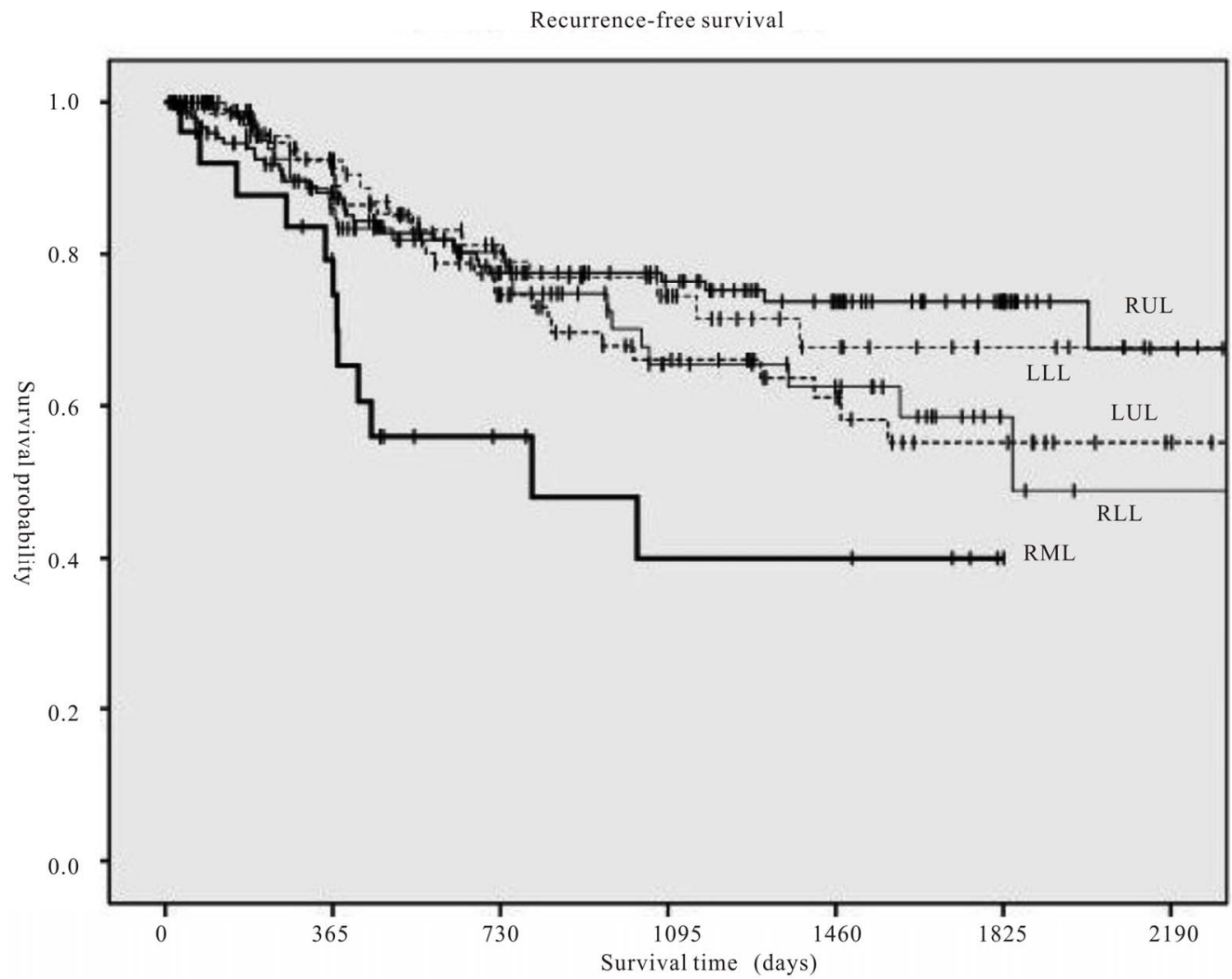

(a)

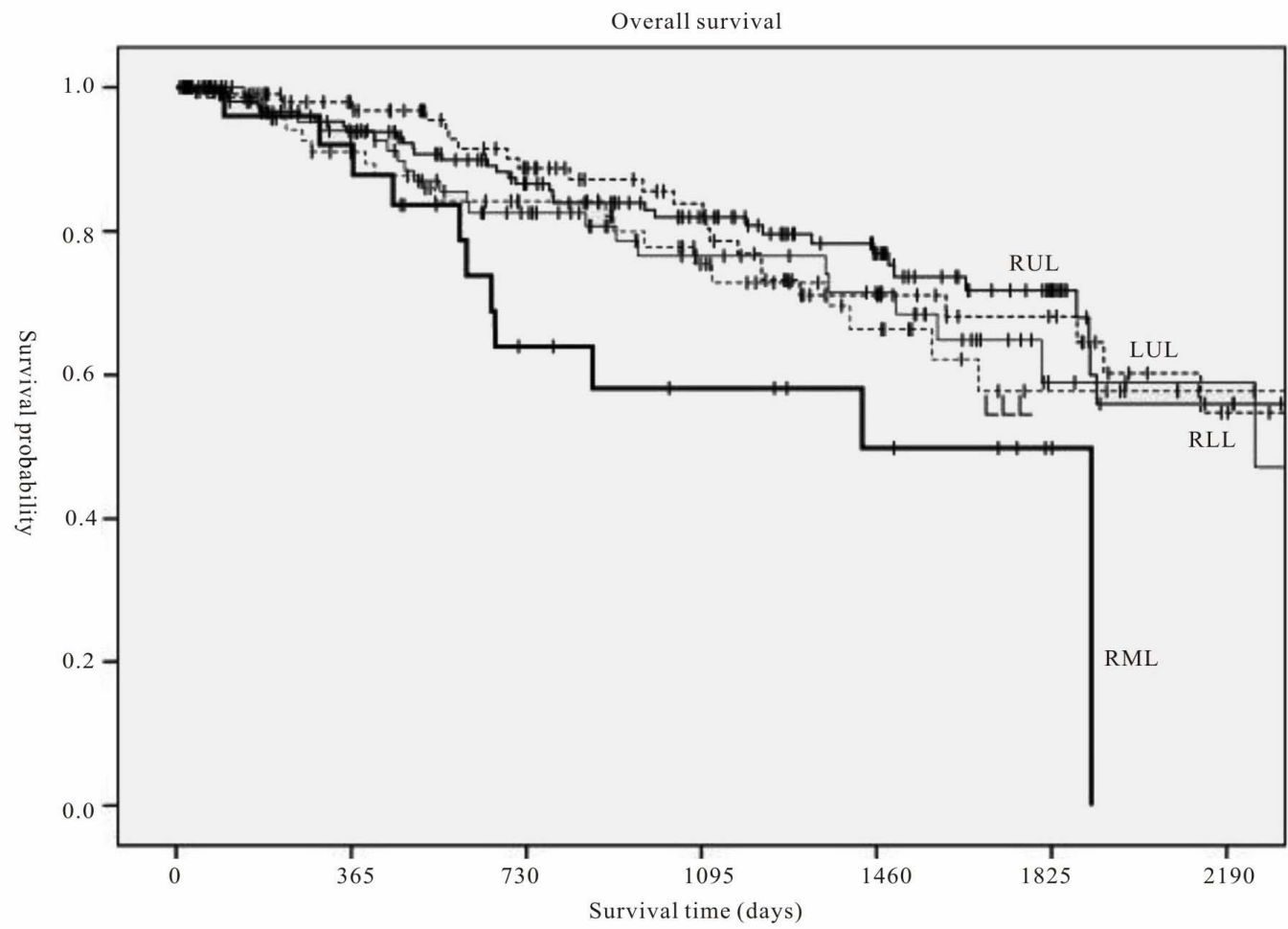

(b)

Figure 1. (a) Kaplan-Meier curve for recurrence-free survival according to the location, log-rank p = 0.018; (b) Kaplan-Meier curve for overall survival according to the location, $\log -$ rank $p=0.109$. 
stump. In 1996, Miura et al. also described that the rate of exploratory thoracotomy was higher for RML cancer than for the cancer of the other lobes ( $16.5 \%$ vs. $7.3 \%)$. 3) We also demonstrated that significant association of the cancer located in the RML was observed with the high rate of lymph node metastasis and the high rate of locoregional recurrence. In 1999, Asamura et al. described that the rate of multiple-station metastasis was higher for RML cancer than for the cancer of the other lobes. 4) Over the last couple of decades, numerous advances have been made for early diagnosis of lung cancer, such as high-resolution CT (HRCT) and 18-fluoro-deoxyglucose positron emission tomography (FDG-PET), however, the rate of incomplete resection and lymph node metastasis of cancer located in the RML was still high in this study. Therefore, we believe that primary lung cancer located in the RML still has a poor prognosis because of the high risk of incomplete resection and the high rate of lymph node metastasis associated with this cancer.

The limitations of this study were as follows: 1) the study was retrospective in nature; 2) the size of the patients with lung cancer located in the RML was small; 3 ) routine adjuvant chemotherapy for stage IB-IIIA was started in 2006; 4) routine FDG-PET for staging was started in 2007. Therefore, in this study, the role of adjuvant chemotherapy or FDG-PET remains uncertain.

In conclusion, despite recent advances in the diagnosis and treatment of lung cancer, the rate of incomplete resection was higher for cancer located in the RML as compared with that for cancer arising from other lobes, evaluation of complete resection was revealed that the outcome for RML cancer was worse than that for cancer arising from other lobes. Further early diagnosis and ad- juvant therapy are needed for improving the prognosis of cancer located in the RML.

\section{REFERENCES}

[1] J. H. Gifford and J. K. Waddington, "Review of 464 Cases of Carcinoma of Lung Treated by Resection," British Medical Journal, Vol. 1, No. 5021, 1957, pp. 723730. doi:10.1136/bmj.1.5021.723

[2] R. G. Vincent, H. Takita, W. W. Lane, A. C. Gutierrez and J. W. Pickren, "Surgical Therapy of Lung Cancer," The Journal of Thoracic and Cardiovascular Surgery, Vol. 71, No. 4, 1976, pp. 581-591.

[3] G. Freise, A. Gabler and S. Liebig, "Bronchial Carcinoma and Long-Term Survival. Retrospective Study of 433 Patients Who Underwent Resection," Thorax, Vol. 33, No. 2, 1978, pp. 228-234. doi:10.1136/thx.33.2.228

[4] H. Miura, H. Kato, C. Konaka, J. Usuda, O. Uchida and O. Taira, "Primary Lung Cancer of the Middle Lobe. Is Its Prognosis Poor?" Lung Cancer, Vol. 14, No. 2-3, 1996, pp. 273-279. doi:10.1016/0169-5002(96)00553-3

[5] H. Asamura, H. Nakayama, H. Kondo, R. Tsuchiya and T. Naruke, "Lobe-Specific Extent of Systematic Lymph Node Dissection for Non-Small Cell Lung Carcinomas According to a Retrospective Study of Metastasis and Prognosis," The Journal of Thoracic and Cardiovascular Surgery, Vol. 117, No. 6, 1999, pp. 1102-1111. doi:10.1016/S0022-5223(99)70246-1

[6] Y. Sakao, S. Okumura, M. Mingyon, H. Uehara, Y. Ishikawa and K. Nakagawa, "The Impact of Superior Mediastinal Lymph Node Metastases on Prognosis in Non-Small Cell Lung Cancer Located in the Right Middle Lobe," Journal of Thoracic Oncology, Vol. 6, No. 3, 2011, pp. 494-499. doi:10.1097/JTO.0b013e31820b8891 